\title{
A Complementary Cyber Swarm Algorithm
}

\author{
Peng-Yeng Yin, National Chi Nan University, Taiwan \\ Fred Glover, OptTek Systems, Inc., USA \\ Manuel Laguna, University of Colorado, USA \\ Jia-Xian Zhu, National Chi Nan University, Taiwan
}

\begin{abstract}
A recent study (Yin et al., 2010) showed that combining particle swarm optimization (PSO) with the strategies of scatter search (SS) and path relinking (PR) produces a Cyber Swarm Algorithm that creates a more effective form of PSO than methods that do not incorporate such mechanisms. This paper proposes a Complementary Cyber Swarm Algorithm (C/CyberSA) that performs in the sameleague as the original Cyber Swarm Algorithm but adopts different sets of ideas from the tabu search (TS) and the SS/PR template. The C/CyberSA exploits the guidance information and restriction information produced in the history of swarm search and the manipulation of adaptive memory. Responsive strategies using long term memory and path relinking implementations are proposed that make use of critical events encountered in the search. Experimental results with a large set of challenging test functions show that the C/CyberSA outperforms two recently proposed swarm-based methods by finding more optimal solutions while simultaneously using a smaller number of function evaluations. The C/CyberSA approach further produces improvements comparable to those obtained by the original CyberSA in relation to the Standard PSO 2007 method (Clerc, 2008).
\end{abstract}

Keywords: $\quad$ Guiding Points, Multi-Start, Particle Swarm Optimization, Scatter Search, Tabu Restriction

\section{INTRODUCTION}

Metaheuristics are master strategies that guide and modify slave heuristics to produce solutions beyond those that are normally generated for local optimality. Effective metaheuristics make a search plan of intensification and diversification to reach a good trade-off between solution quality and computational effort. Intensification exploits information about elite solutions that

DOI: $10.4018 /$ jsir.2011040102 were previously found as a basis for focusing the search in regions anticipated to harbor additional solutions of high quality. Diversification promotes the exploration of regions appreciably different from those previously examined in order to produce new solutions with characteristics that depart from those already seen. Intensification and diversification work together to identify new promising regions when the slave heuristics stagnate in the executed search courses. Many intelligent algorithms fall in the territory of metaheuristics. 
Some exemplary algorithms (Luke, 2009) are genetic algorithms (GA), simulated annealing (SA), ant colony optimization (ACO), tabu search (TS), particle swarm optimization (PSO), scatter search (SS), greedy randomized adaptive search procedure (GRASP), variable neighborhood search (VNS), to name a few. A recent survey and descriptive analysis of metaheuristic algorithms can be found in Sorensen and Glover (2010).

Slave heuristics embedded in metaheuristic methods often adopt solution combination or neighborhood exploration processes to generate new solutions based on the current state of search. Solution combination approaches produce new solutions by exchanging information between candidate solutions (for example, crossover operation executed in GA) or by using candidate solutions as guiding points for producing new solutions (for example, by reference to the best experiences in PSO or the path relinking (PR) process used in SS). Alternatively, neighborhood exploration employs incremental changes, called moves, which progress from one solution to another within local regions called neighborhoods that are considered relevant to the search (as by changing one or a small number of elements within a current solution). Sophisticated neighborhood concepts like ejection chains (Glover, 1996b; Rego \& Glover, 2009) have been proposed for tackling a variety of complex problems and various types of multiple neighborhood strategies (Glover, 1996a; Mladenovic \& Hansen, 1997; Sörensen, Sevaux, \& Schittekat, 2008; Lu, Hao, \& Glover, 2010) have been proposed for enriching the set of moves employed during the search. To avoid reversing the search course and prevent getting trapped in local optima, some solution attributes and move directions may be forbidden by means of tabu restrictions as proposed in tabu search, or multi-start mechanisms may be employed to initiate a new search thread in an uncharted region. There are other metaheuristic methods employing gradient-based or derivative-free supplementary procedures. The best of these methods are provided by Hedar and Fukushima (2006) and Duarte et al. (2007) for problems of moderate dimension, and by Hvattum and Glover (2009) and Vaz and Vicente (2007) for problems of large dimension. Duarte et al. (2011b) analyzes the performance of two path relinking variants: the static and the evolutionary path relinking. Both are based on the strategy of creating trajectories of moves passing through high quality solutions in order to incorporate their attributes to the explored solutions.

Methods that employ both solution combination and neighborhood exploration perform more effectively by carefully coordinating the slave heuristics with the master strategy employed. An illustration of this is provided, for example, by methods that integrate tabu search with classical direct search for global function optimization. Chelouah and Siarry (2005) proposed a continuous tabu simplex search (CTSS) method that uses the Nelder-Mead simplex algorithm to accelerate convergence towards a minimum within a detected promising region, while maintaining the tabu search (TS) restriction as a mechanism to search uncharted solution space. Hedar and Fukushima (2006) introduced a directed TS (DTS) method for non-linear global optimization which, instead of identifying promising regions before the application, immediately applies the Nelder-Mead method at every non-improving trial point obtained by the TS neighborhood search. A study of larger problems by Hvattum and Glover (2009) shows that the use of direct search methods different from the Nelder-Mead procedure can produce superior results.

Another approach (Nakano et al., 2007; Shen et al., 2008; Wang et al., 2007) akin to this research direction is the hybridization of PSO and TS. The TS processes for managing adaptive memory via responsive strategies enables PSO to implement intensification and diversification searches more effectively. On the one hand, the attribute values which produced high quality solutions in TS recency memory can be reserved for other particles in the future. On the other hand, less fit attribute values contained in the adaptive memory are designated as tabu-active and the particles are pulled away from these attribute values. The 
particle swarm and pattern search method (Vas and Vicente, 2007) referred to as PSwarm is a pattern search method incorporating particle swarm search as a step within its framework. In additional to the original mesh search, the particle swarm search can explore the nonconvexity of the objective function. More recently, Yin et al. (2010) introduced a Cyber Swarm Algorithm (CyberSA) as the marriage of PSO and the scatter search/path relinking (SS/PR) template that obtains improved outcomes. With the addition of an external memory, embodied in a reference set consisting of the best solutions observed throughout the evolution history, useful information is produced and maintained that is not attainable by relying on traditional PSO mechanisms involving particle experiences.

We propose a restricted variant of the CyberSA by strategically exploring special guidance and restriction information. The contributions of this work are: (1) the augmentation of the search capability of CyberSA by considering additional ideas from TS and SS/ PR, (2) a multi-level (short term, middle term, and long term) memory manipulations designed to reinforce the search process, and (3) extensive performance evaluation of the proposed method with a large set of diverse benchmark functions.

The remainder of this paper is organized as follows. Section 2 presents a literature review of fundamental PSO methods relevant to our work. Section 3 proposes the Complementary Variant of the Cyber Swarm Algorithm and describes its salient features. Section 4 presents experimental results together with an analysis of their implications. Finally, concluding remarks and discussions are given in Section 5.

\section{PSO LITERATURE REVIEW}

The introduction of particle swarm optimization (PSO) has motivated many researchers to develop various swarm algorithms by drawing fruitful notions from other domains. These development efforts include two main types: the exploitation of guidance information and the hybridization with other intelligent search strategies.

\subsection{Exploitation of Guidance Information}

The PSO proposed by Kennedy and Eberhart (1995) has exhibited effectiveness and robustness in many applications, such as evolving artificial neural networks (Eberhart \& Shi, 1998), reactive power and voltage control (Yoshida \& Kawata, 1999), state estimation for electric power distribution systems (Shigenori et al., 2003), and image compression (Feng et al., 2007). PSO has drawn on a sociocognition model to gain recognition as a useful global optimizer. A swarm of particles is assumed to follow the social norm manifest of convergent behaviors. The social norm consists of quality experiences by comparing search trajectories of individual particles. This is an auto-catalytic process that the social norm influences the individual behaviors which in turn collectively improve the social norm.

The original version of PSO is quite simple. A swarm of particles iteratively update their positional vectors by reference to previous positions and two forms of the best trajectory experience, namely, the personal best (pbest) and the global best (gbest). The personal best is the best position that a particle has experienced, while the global best is the best position ever experienced by the particles in the swarm. Let the optimization problem be formulated with $R$ decision variables and a particle $P_{i}=\left(p_{i 1}, p_{i 2}\right.$, $\left.\ldots, p_{i R}\right)$ representing a candidate solution whose search trajectory is iteratively determined by adding a velocity vector $V_{i}=\left(v_{i 1}, v_{i 2}, \ldots, v_{i R}\right)$ to its previous position. According to Clerc's Stagnation Analysis (Clerc \& Kennedy, 2002), the convergence of the particles' trajectories is mathematically guaranteed by the following equations.

$$
\begin{aligned}
& v_{i j} \leftarrow K\left(v_{i j}+\phi_{1} \text { rand }_{1}\left(\text { pbest }_{i j}-p_{i j}\right)+\phi_{2}\right. \\
& \left.\operatorname{rand}_{2}\left(\text { gbest }_{j}-p_{i j}\right)\right)
\end{aligned}
$$




$$
K=\frac{2}{\left|2-\left(\phi_{1}+\phi_{2}\right)-\sqrt{\left(\phi_{1}+\phi_{2}\right)^{2}-4\left(\phi_{1}+\phi_{2}\right)}\right|}
$$

(with the constraint $\varphi_{1}+\varphi_{2}>4$ )

and

$$
p_{i j} \leftarrow p_{i j}+v_{i j}
$$

where $\varphi_{1}$ and $\varphi_{2}$ are the cognitive coefficients, rand $_{1}$ and rand $_{2}$ are random real numbers drawn from $U(0,1)$, and $K$ is the constriction coefficient. In essence, the particle explores a potential region defined by pbest and gbest, while the cognitive coefficients and the random multipliers change the weightings for the two best solutions in every iteration.

As indicated by the term "small world" in sociology, two people indirectly share information via the social network. The speed on which the information spreads depends upon the structure of the social network. Kennedy (1999) has studied the effects of neighborhood topologies on particle swarm performance. The local best leader (lbest), the best position visited by any member in the neighborhood of a designated given particle, is used as a substitute for gbest. He found that the best neighborhood topology depends on the problem context. The Star topology (the gbest version where each particle is connected to all particles in the swarm) spreads the individual information throughout the swarm most quickly and can expedite the convergence of explored solutions in unimodal function optimization problems. However, the Ring topology (each particle is connected to exactly two other particles) can usually produce better solutions on multimodal functions than the Star topology because the former structure postpones the information transmission between two arbitrary particles and is more effective in avoiding being trapped in local optimality. The performance of other topologies, such as Wheel or Pyramid, varies from problem to problem.

Miranda et al. (2007) proposed a Stochastic Star topology where a particle is informed by gbest with a predefined probability $p$. Their experimental results showed that the Stochas- tic Star topology leads in many cases to better results than the original Star topology. The standard PSO 2007 (Clerc, 2008) regenerates a random permutation of particles before each iteration. Hence, the resulting neighborhood structure is in essence a random topology.

While most of the PSO algorithm variants conduct the swarm evolution using two particle leaders, Clerc's Stagnation Analysis (Clerc \& Kennedy, 2002) does not limit consideration to two cognitive coefficients, but only requires that the parts sum to a value that is appropriate for the constriction coefficient $K$. Mendes et al. (2004) have proposed to combine all neighbors' information instead of only using lbest. Let $\Omega_{i}$ be the set of neighbors' indices of particle $i$ and let $\omega_{k}$ be an estimate of the relevance of particle $k$ as being an informant of particle $i$. Then the velocity can be updated by:

$v_{i j} \leftarrow K\left(v_{i j}+\phi\left(\right.\right.$ mbest $\left.\left._{i j}-p_{i j}\right)\right)$

and:

$\begin{aligned} \text { mbest }_{i j} & =\frac{\sum_{k \in \Omega_{i}} \omega_{k} \phi_{k} \text { pbest }_{k j}}{\sum_{k \in \Omega_{i}} \omega_{k} \phi_{k}}, \phi=\sum_{k \in \Omega_{i}} \phi_{k} \\ \text { and } \phi_{k} & \in U\left[0, \frac{\phi_{\max }}{\left|\Omega_{i}\right|}\right]\end{aligned}$

As all the neighbors contribute to the velocity update, the focal particle is fully informed.

The Cyber Swarm Algorithm (CyberSA), which has produced better results than the standard PSO 2007 method, uses three leaders in the velocity adjustment and extends the neighborhood topology by additionally including the reference set construction of the SS/PR template. The reference set is not restricted simply to neighbors, but consists of the best solutions observed throughout the evolution history. The CyberSA reinstates pbest and gbest in the group ofleaders and systematically selects each member from the reference set as the third leader. Additional details of this method are discussed subsequently. 


\subsection{Hybridization with Outsource Strategies}

Researchers have proposed that PSO may be extended by taking into account useful strategies from other methodologies. The obvious advantage of hybridizing PSO is the potential for enhancing the intensification/diversification synergy and improving the regions selected to be explored in the course of the search. Often, hybrid algorithms exhibit better performance when solving complex problems, such as the optimization of difficult multimodal functions. These mechanisms are mainly found within the framework of evolutionary algorithms and adaptive memory programming concepts derived from tabu search.

In the hybrid algorithm proposed by Angeline (1999) particles with low fitness are replaced by those with high fitness using natural selection while these particles preserve their original best experience. This approach is reported to facilitate the exploration of highly promising regions while maintaining experience diversity. Lovbjerg et al. (2001) implement a hybrid approach by inserting a breeding (recombination) step after the movement of all particles. Particles are selected with equal probability to become parents and in turn are replaced by offspring that are generated using the arithmetic crossover operator. In order to keep diversity in the gene pool, particles are divided into subpopulations and the breeding is allowed both within a given subpopulation and between different subpopulations.

A hybridization of generalized pattern search (Audet \& Dennis, 2003) with PSO is proposed by Vas and Vicente (2007) to produce the PSwarm algorithm that consists of alternating iterations of search and poll steps. The search enforces a step of particle swarm search (and expands the mesh size parameter) if such a step causes the individual bests of the particles to improve. Otherwise, the poll step is activated, consisting of applying one step of local pattern search (along the canonical mesh) on the best particle of the entire swarm. The mesh size parameter is either expanded or contracted according to whether the pattern search improves the best particle. PSwarm has been shown to outperform several optimizers on an experiment over a set of 122 benchmark functions.

A number of effective hybrids have been produced by incorporating various elements of tabu search. Nakano et al. (2007) divide the particles into two sub-swarms that play the roles of intensification and diversification, respectively. When an attribute value in the global best solution is not updated for a number of iterations, the attribute value is designated as tabu. The particles from the intensification sub-swarm fix the attribute values as specified by the tabu restrictions and contained in the global best solution, while the particles in the diversification sub-swarm are encouraged to pull away from solutions containing the tabu attributes. Shen et al. (2008) propose an approach called HPSOTS which enables the PSO to leap over local optima by restraining the particle movement based on the use of tabu conditions. Wang et al. (2007) enhances the diversification capability of PSO by setting the less fit attributes contained in the global best solution as tabu-active and repelling the particles from the tabu area.

The Cyber Swarm Algorithm (CyberSA) of Yin et al. (2010) creates an enhanced form of swarm algorithms by incorporating three features: (1) augmenting the information sharing among particles by learning from the reference set members, (2) systematically generating dynamic social networks in order to choose various solutions as the leaders such that the search can adapt to different functional landscape, and (3) executing diversification strategies based on path relinking approaches as a response to the status of the adaptive memory. The success of this method has motivated us to examine another variant that draws on alternative ideas from the same sources. 


\section{COMPLEMENTARY VARIANT OF CYBER SWARM ALGORITHM}

As disclosed in the literature discussed in Section 2, the performance of PSO can be improved by exploiting the guidance information and hybridizing the method with outsource strategies. Following this theme, the CyberSAcreates an effective form of PSO by carefully selecting leading solutions and embedding scatter search/path relinking (SS/PR) strategies. The Complementary Cyber Swarm Algorithm (C/ CyberSA) proposed here uses different sets of ideas from the adaptive memory programming perspective of tabu search and scatter search. As we show, the C/CyberSA can produce improvements comparable to those obtained by the original CyberSA in relation to those PSO methods that do not incorporate adaptive memory programming ideas.

\subsection{Using Guidance Information}

As previously noted, the choice of neighborhood topologies and leading solutions significantly affects the particle swarm performance. The literature discloses that the use of a dynamic neighborhood (Miranda et al., 2007; Clerc, 2008; Yin et al., 2010) and the local best solution lbest (Kennedy, 1999; Clerc, 2008) leads to a better performance. These notions create a form of multiple neighborhood search in which the neighboring particles (each maintaining a search trajectory) are selected at random or systematically and the local optimum corresponds to the best solution encountered by the multiple search trajectories.

Our proposed C/CyberSA method generates a random permutation of particles on a Ring topology before performing each iteration, so the neighboring particles are very likely different from those assigned at previous iterations. The three leaders, the local best solution (lbest) observed by the neighboring particles, the overall best solution (gbest) found by the entire swarm, and the individual best experience (pbest) for the operating particle, are used as the guiding solutions. More precisely, the $\mathrm{C} /$ CyberSA uses the following velocity updating formula for the $i$ th particle,

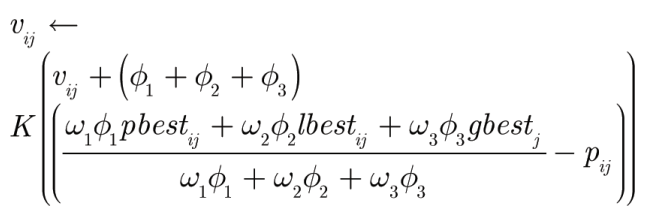

The weight $\omega_{i}$ is selected to be the same for each of the three guiding solutions.

The advantage of using three guiding solutions has been empirically verified in several relevant studies. For example, Campos et al. (2001) found that in scatter search most of the high quality solutions come from combinations using at most 3 reference solutions. Mendes et al. (2004) have also more recently found that their FIPS algorithm (which treats the previous bests of all neighbors as guiding solutions) performs best when a neighborhood size of 2 to 4 neighbors is used, and increasing the neighborhood size causes the overall performance to deteriorate. The CyberSA method also achieves particularly good outcomes when using three strategically selected guiding solutions.

\subsection{Using Restriction Information}

The adaptive memory programming perspective of tabu search provides a fruitful basis for generating incentives and restrictions to guide the search towards more promising regions. The central idea is to compare previous states (e.g., selected solution attributes) stored in the adaptive memory to those states of new candidate solutions currently contemplated.

Our C/CyberSA approach uses multiple levels of adaptive memory to exploit the benefits of restriction information. Three categories of adaptive memory are employed: (1) a shortterm memory (STM) that records the solutions visited by individual particles within a short span of recent history; (2) a middle-term memory (MTM) that tallies the solutions that pass a certain acceptance threshold dynami- 
cally changed according to the current state; and (3) a long-term memory (LTM) that tracks the frequency or duration of critical events and activates appropriate reactions. Immediately following, we present the implementation for STM and MTM, and the description for LTM is given in Subsection 3.3.

In PSO, a swarm of particles construct their individual search courses to accumulate rewarded experiences and there is no additional value in allowing particles to be transformed into recent previous solutions. We therefore use STM to prevent individual particles from reversing recent moves as they undergo the transformation to produce new particles. The notion of a "tabu ball" proposed by Chelouah and Siarry (2000) is adopted in our implementation. When a particle is replaced by a new solution based on Eq. (6), the solution is designated as tabuactive with a tabu tenure. A tabu ball centered at this solution with radius $r$ is created and prohibits the acceptance of new solutions produced inside this ball during its tabu tenure. Let the set of centers of currently STM active tabu balls be $\pi_{S T M}=\left\{s_{1}, s_{2}, \ldots, s_{T}\right\}$, a new solution $s^{\prime}$ is rejected (tabu) if it is contained within any of the tabu balls, i.e., $\left\|s^{\prime}-s_{i}\right\| \leq r, \forall s_{i} \in \pi_{S T M}$, where $\|\cdot\|$ denotes the Euclidean norm (additional ways to generate "tabu regions" along with the possibility to use other distance norms are proposed in Glover, 1994). However, the tabu restriction can be overruled if $s^{\prime}$ meets some aspiration criterion. The Aspiration_by_objective rule stipulates that a tabu solution $s^{\prime}$ can be accepted if its objective value is better than that of the overall best solution. To introduce a form of vigor into the search that accommodates varying widths of local minima, the tabu tenure of a tabu ball is determined dynamically and drawn randomly from a pre-specified range, motivated by the fact that a dynamic tabu tenure typically performs better than a constant tabu tenure in TS implementations. To finely tune the size of the tabu ball in accordance with different phases of the search, the ball radius $r$ is reduced by a ratio $\beta$ upon the detection of stagnation as noted in the next subsection.

For the implementation of tabu restrictions with MTM, we prevent a particle from updating its personal best (pbest) if the candidate solution is too close to the recent best of any particle. Again, a tabu ball is created for a newly produced pbest solution and the update of pbest is prohibited for any particle inside this tabu ball during its tabu tenure. Denote the set of centers of currently MTM active tabu balls by $\pi_{M T M}$. The MTM incorporates the same rules for the aspiration criterion, dynamic tabu tenure, and tabu ball radius reduction as enforced in the STM mechanism.

We do not impose a tabu restriction on the update of the overall best (gbest) because it is always beneficial to obtain a better overall best solution during the search (and such a solution automatically satisfies the aspiration criterion in any case).

\subsection{Responsive Strategies}

Longer term strategies responding to detections of critical changes in LTM are invoked when a short term strategy has lost its search efficacy. Successful applications of this principle have been seen in both static and dynamic optimization problems (James et al., 2009; Lepagnot et al., 2010), reinforcing the supposition that promising solutions having features differing from those previously seen are likely to be obtained by using longer term strategies.

One of the effective longer term strategies is path relinking (PR). (For recent surveys, see Ho \& Gendreau, 2006; Rego \& Glover, 2008.) PR is a search process which constructs a link between two or more strategically selected solutions. The construction starts with one solution (called the initiating solution) and moves to or beyond a second solution (referred to as the guiding solution). PR transforms the initiating solution into the guiding solution by generating moves that successively replace an attribute of the initiating solution that is contained in the guiding solution. The link can be constructed in 
both directions by interchanging the roles of the initiating and guiding solutions, or can proceed from both ends toward the middle.

PR can emphasize either intensification or diversification. For example, intensification strategies may choose reference solutions to be the best solutions encountered in a common region while diversification strategies can select reference solutions that lie within different regions. Reference solutions are selected according to their status in the LTM and the appropriate PR strategies are triggered upon the detections of critical events. We propose two responsive PR strategies and the corresponding triggering critical events in the following.

(1) Diversification PR strategy - particle restarting: The C/CyberSAmanages multiple particle search threads exploring promising regions in the solution space. The identified promising regions are located by using pbest which in turn is used to identify the particles to exploit these regions. A critical event arises when pbest has not improved for $t_{2}$ successive search iterations. This indicates the region has been overexploited and the corresponding particle should be repositioned in an uncharted region to start a new round of search. In light of this, PR can focus on identifying new promising regions. We do this by selecting two reference solutions from two under-exploited regions, using the biased random approach proposed in the original CyberSAmethod. This approach generates a biased-random solution which is likely to be in a position at a maximal distance from all previous trial solutions. Let the two reference solutions be RandSol $_{1}$ and $\mathrm{RandSol}_{2}$, respectively. The diversification PR strategy is triggered by the critical event to construct a link PR ( RandSol, RandSol $_{2}$ ) between the two reference solutions. The best solution observed over the constructed link is designated as the initiating particle for restarting a new search thread. It is noted that although the particle has been repositioned, the content of the adaptive memory (such as pbest, gbest, tabu list, tabu tenure, and tabu ball radius) is retained.

The diversification PR strategy not only diversifies the search (by creating a new thread that has not been targeted before) but also tunnels through different regions that have contrasting features. This strategy is designed based on the anticipation that the solutions with fruitful information for the current search state are those contained in uncharted regions or near the boundaries between regions having contrasting features.

(2) Intensification PR strategy-swarm shrinking: The other critical event is the detection of swarm stagnation. This happens when the distributed particles have exhausted their search efficacy and the swarm overall best solution gbest has not improved for $t_{1}$ successive search iterations. We propose an intensification PR strategy named swarm shrinking which regenerates a new swarm within the neighborhood proximity of gbest to replace the original population of particles in order to intensify the search in the overall best region. Each particle in the new swarm is generated by applying a truncated PR process, Truncated_PR(gbest, Rand$\mathrm{Sol}_{1}$ ), using gbest as the initiating solution and, again, employing a solution RandSol produced by the biased random approach as the guiding solution. The truncated PR process starting with the initiating solution performs a few moves and only constructs a partial link. In our implementation, we terminate the link construction when one tenth of the number of attributes in gbest have been replaced (at least one attribute has been replaced if gbest has less than 10 attributes). The best solution observed on the partial link is used to replace the original particle.

In the process of swarm shrinking, all the elements excluding the tabu ball radius in the 
adaptive memory hold their original values. The length of the tabu ball radius is halved to facilitate a finer search with the shrunken swarm.

\subsection{C/CyberSA Pseudo Code}

The C/CyberSA design is elaborated in the pseudo codes shown in Figure 1. The three important features (guidance information, restriction information, and responsive strategies) of the $\mathrm{C} / \mathrm{CyberSA}$ are in boldface to emphasize these features within the algorithm. In the initialization phase (Step 1) the initial values for particle positions and velocities and the values for the elements (pbest, gbest, tabu ball radius, etc.) stored in the adaptive memory are given. In the main-loop iterations (Step 2), the swarm conducts its search using these three components. To generate a dynamic neighborhood, the particles are randomly arranged on a Ring topology. Each particle moves with the guidance information provided by pbest , $_{\text {, }}$ best $t_{i}$ and gbest (Step 2.2.1). However, any movement leading into a tabu ball stored in STM is prohibited (Step 2.2.3). The individual pbest $t_{i}$ is updated if a better solution is produced by the movement which is not tabu by the MTM restriction (Step 2.2.4). Finally, as shown in Step 2.4, the intensification PR strategy is executed if gbest has not improved for a number of standing iterations. Otherwise, the diversification strategy is performed if a particular pbest ${ }_{i}$ stagnates during the attempted improvement process.

\section{EXPERIMENTAL RESULTS AND ANALYSIS}

We have conducted extensive experiments to evaluate the performance of the C/CyberSA. The experimental results disclose several interesting outcomes in addition to establishing the effectiveness of the proposed method. The platform for conducting the experiments is a PC with a $1.8 \mathrm{GHz}$ CPU and 1.0 GB RAM. All programs are coded in $\mathrm{C}++$ language.

\subsection{Performance Measures and Competing Algorithms}

We measure the performance of competing algorithms in terms of effectiveness and efficiency. Effectiveness measures how close the quality of the obtained solution is to that of the optimal solution while efficiency assesses how fast a given algorithm can obtain a solution with a target quality.

The effectiveness measure is gauged by reference to the best objective value obtained by a competing algorithm that has been allowed to consume a maximum number of function evaluations equal to 160,000 . (We selected this number because we observed the competing algorithms converge for most of the test functions after performing 160,000 evaluations.) When comparing the effectiveness of a target algorithm against a reference algorithm, a relative measure called merit is often used and defined as merit $=\left(f_{p}-f^{*}+\varepsilon\right) /\left(f_{q}-f^{*}+\varepsilon\right)$, where $f_{p}$ and $f_{q}$ are the mean best objective value obtained by the target algorithm and the reference algorithm, respectively, $\mathrm{f}^{*}$ is the known global optimum of the test function, and $\varepsilon$ is a small constant equal to $5 \times 10^{-7}$. Without loss of generality, we consider all the test functions to involve minimization, and stipulate that the target algorithm outperforms the reference algorithm if the value of merit is less than 1.0 (where smaller values represent greater differences in favor of the target algorithm).

We employ the policy widely adopted in the literature of representing the efficiency measure as the mean number of function evaluations required by a given algorithm in order to obtain an objective value that is sufficiently close to the known global optimum by reference to a specified gap.

Yin et al. (2010) have shown the advantages of the original CyberSA by comparing it with several other metaheuristics such as the Standard PSO 2007 (Clerc, 2008), C-GRASP (Hirsch etal., 2007), Direct Tabu Search(Hedar 


\section{Figure 1. Pseudo codes for the Complementary Cyber Swarm Algorithm (C/CyberSA)}

1 Initialize.

1.1 Generate $N$ particle solutions, $P_{i}=\left(p_{i 1}, p_{i 2}, \ldots, p_{i R}\right), 1 \leq i \leq N$, at random.

1.2 Generate $N$ velocity vectors, $V_{i}=\left(v_{i 1}, v_{i 2}, \ldots, v_{i R}\right), 1 \leq i \leq N$, at random.

1.3 Evaluate the fitness of each particle fitness $\left(P_{i}\right)$, and set previous best solution pbest $t_{i}$ to $P_{i}$. Determine the overall best solution gbest as the best among all pbest.

1.4 Set initial tabu ball radius $r$

2 Repeat until a stopping criterion is met.

2.1 Generate a random permutation of particles on a Ring topology to determine local bests $l b e s t_{i}, \forall i=1, \ldots, N$.

2.2 For each particle $P_{i}, \forall i=1, \ldots, N$, Do

2.2.1 Guidance: Compute the velocity using the three strategically selected guiding solutions.

$$
v_{i j} \leftarrow K\left(v_{i j}+\left(\varphi_{1}+\varphi_{2}+\varphi_{3}\right)\left(\frac{\omega_{1} \varphi_{1} \text { pbest }_{i j}+\omega_{2} \varphi_{2} \text { lbest }_{i j}+\omega_{3} \varphi_{3} \text { gbest }_{j}}{\omega_{1} \varphi_{1}+\omega_{2} \varphi_{2}+\omega_{3} \varphi_{3}}-p_{i j}\right)\right)
$$

2.2.2 Compute the tentative movement $P_{i}^{\prime}=P_{i}+V_{i}$.

2.2.3 STM restriction: If $\left\|P_{i}^{\prime}-s_{i}\right\| \leq r, \forall s_{i} \in \pi_{S T M}$ and $P_{i}^{\prime}$ does not satisfy the aspiration criterion, goto Step 2.2.1.

2.2.4 MTM restriction: If $P_{i}^{\prime}$ is better than $p b e s t_{i}$ and $\left\|P_{i}^{\prime}-s_{i}\right\|>r, \forall s_{i} \in \pi_{M T M}$, or $P_{i}^{\prime}$ satisfies the aspiration criterion, pbest $_{i} \leftarrow P_{i}^{\prime}$

2.3 Determine the overall best solution $g b e s t$ as the best among all pbest $t_{i}, \forall i=1, \ldots, N$.

2.4 Intensification PR strategy (swarm shrinking): If gbest has not improved for $t_{l}$ iterations, reinitiate all particles and halve the tabu ball radius by

$P_{i} \leftarrow$ Truncated_PR(gbest, RandSol $\left._{1}\right), \forall i=1, \ldots, N$

$r \leftarrow 0.5 r$

Diversification PR strategy (particle restarting): Else if a particular pbest $_{i}$ has not improved for $t_{2}$ iterations, replace its particle by $P_{i} \leftarrow P R\left(\right.$ RandSol $_{1}$, RandSol $\left._{2}\right)$

\& Fukushima, 2006), Scatter Search (Laguna \& Marti, 2005), and Hybrid Scatter Tabu Search (Duarte etal., 2011a). In our present comparison we also include the PSwarm algorithm of Vaz and Vicente (2007), which embeds the swarm algorithm into the pattern search framework.

The parameter values used by the $\mathrm{C} /$ CyberSA have been determined based on preliminary experiments with a variety of test values, which led us to select the following settings. The size of the swarm is set to consist of 40 particles. At most five trial particles are produced by each "particle move" operation. The first non-tabu trial particle is accepted to be the next position of the focal particle, and the new position is marked as tabu with a dynamic tenure drawing a random value from the range $[5,15]$. If all the five trial particles are tabu, the default aspiration chooses the one with the shortest tabu tenure to be released and accessed. The radius of the tabu ball is initialized to one percent of the mean range of variable values.
The responsive longer term strategies (swarm shrinking and particle restarting) are executed when critical events are observed with the parameter settings of $t_{1}=100$ and $t_{2}=200$. As for the other competing algorithms, the parameter values are set to the suggested values according to their original papers.

\subsection{Performance}

\subsubsection{Experiment 1}

Our first experiment evaluates the effectiveness of the C/CyberSA with a set of 30 test functions that are widely used in the literature (Laguna \& Marti, 2003; Hedar \& Fukushima, 2006; Hirsch et al., 2007; Yin et al., 2010). All these functions are continuous and together they present a wide variety of different landscapes. A hundred repetitive runs are executed for each of the three methods compared: the Standard PSO 2007, CyberSA, and C/CyberSA. Each run of 
a given algorithm is terminated when 160,000 function evaluations have been exhausted and the best function value obtained is considered as the outcome of this run.

The mean best function value over the 100 independent runs and the merit value relative to the mean best result of the Standard PSO 2007 are shown in Table 1. The numerical values in the parentheses correspond to the standard deviation of the best function values over the 100 repetitions. We observe that, except for the simple functions where all competing algorithms can obtain the global optimum, CyberSA is more effective than the Standard PSO 2007 by being able to obtain a lower mean best function value for the test functions. The product of the merit values for the CyberSA is equal to 1.15E-36. The best function value reported by the CyberSA is significantly closer to the global optimum than that obtained by the Standard PSO 2007.

The C/CyberSA exhibits similar effectiveness as observed from its mean best function value and merit value. The product of the merit values for the C/CyberSA is $1.07 \mathrm{E}-40$ which is even somewhat better than the corresponding product for the CyberSA. However, the $\mathrm{C} / \mathrm{CyberSA}$ is less effective than CyberSA in finding the global optimum for the four functions Shekel(4, 5), Shekel(4, 7), Griewank(20), and Griewank(30). This is compensated by the fact that the C/CyberSA demonstrates significantly greater effectiveness than the CyberSA in tackling the difficult functions like Rosenbrock and Rastrigin having ten or more variables. These findings motivate future investigations of Cyber Swarm methods that combine features of the original CyberSA and the $\mathrm{C} / \mathrm{CyberSA}$ as a basis for creating a method that may embody the best features of both approaches. To get a rough indication of the promise of such an approach, we examined a "trivial combination" of the two methods as follows. Of the eleven functions that are not solved optimally by both CyberSA and $\mathrm{C} / \mathrm{Cy}-$ berSA, we observe that CyberSA obtains better solutions on Shekel(4, 5), Shekel(4, 7), Griewank(20), and Griewank(30), while C/Cy-
berSA obtains better solutions on Rosenbrock(10), Rastrigin(10), Griewank(10), Rosenbrock(20), Rastrigin(20), Rosenbrock(30) and Rastrigin(30). The maximum number of function evaluations required by CyberSA to find its best solution to any of the problems where it performs better is 104,416 , while the maximum number of function evaluations required by $\mathrm{C} / \mathrm{CyberSA}$ to find its best solution to any of the problems where it performs better is 137,504 . Consequently, a "trivial combination" of the two methods that runs CyberSA for 104,416 function evaluations and C/CyberSA for 137,504 function evaluations would yield a method that provides the best solutions on all of these problems within a total number of 241,920 function evaluations. We have allotted a maximum of 250,000 function evaluations for the Standard PSO 2007 as a basis for fair comparison. The resulting version of Standard PSO 2007 does not perform much better than when the method is allotted 160,000 function evaluations, although it can solve Rosenbrock(5) and Zakharov(30) to optimality with 250,000 function evaluations. Consequently, the "trivial combination" of CyberSA and C/CyberSA likewise dominates the Standard PSO 2007 method when the latter is permitted to use 250,000 function evaluations, obtaining better solutions than the Standard PSO method on 16 test problems and matching the Standard PSO 2007 method on the remaining 14 problems. A more sophisticated way of combining the strategies employed by CyberSA and $\mathrm{C} / \mathrm{CyberSA}$ would undoubtedly perform still better, thus reinforcing the motivation for future research to examine ways of integrating the TS and SS/PR strategies embodied in the CyberSA and C/CyberSA methods.

Finally, the value of the standard deviation listed in Table 1 also discloses that the computational results obtained by CyberSA and $\mathrm{C} /$ CyberSA from 100 independent runs are more consistent than those produced by the standard PSO 2007, recommending the use of the CyberSA and C/CyberSA from the worst-case analysis perspective. 
Table 1. Mean best function value with standard deviation and the merit value for the competing algorithms

\begin{tabular}{|c|c|c|c|c|c|c|}
\hline$R$ & $\begin{array}{c}\text { Test } \\
\text { Function }\end{array}$ & $\begin{array}{c}\text { Standard PSO } \\
2007\end{array}$ & CyberSA & $\begin{array}{c}\text { Cy- } \\
\text { berSA } \\
\text { Merit }\end{array}$ & C/CyberSA & $\begin{array}{l}\text { C/Cyber- } \\
\text { SA Merit }\end{array}$ \\
\hline 2 & Easom & $-0.9999(0.0000)$ & $-1.0000(0.0000)$ & 0.3333 & $-1.0000(0.0000)$ & 0.33333 \\
\hline 2 & Shubert & $\begin{array}{l}-186.7202 \\
(0.0071)\end{array}$ & $-186.7309(0.0000)$ & $4.67 \mathrm{E}-5$ & $-186.7309(0.0000)$ & $4.67 \mathrm{E}-5$ \\
\hline 2 & Branin & $0.3979(0.0000)$ & $0.3979(0.0000)$ & 1.0000 & $0.3979(0.0000)$ & 1.0000 \\
\hline 2 & Goldstein-Price & $3.0001(0.0001)$ & $3.0000(0.0000)$ & 0.0050 & $3.0000(0.0000)$ & 0.0050 \\
\hline 2 & Rosenbrock(2) & $0.0000(0.0000)$ & $0.0000(0.0000)$ & 1.0000 & $0.0000(0.0000)$ & 1.0000 \\
\hline 2 & Zakharov(2) & $0.0000(0.0000)$ & $0.0000(0.0000)$ & 1.0000 & $0.0000(0.0000)$ & 1.0000 \\
\hline 3 & De Jong & $0.0000(0.0000)$ & $0.0000(0.0000)$ & 1.0000 & $0.0000(0.0000)$ & 1.0000 \\
\hline 3 & Hartmann(3) & $-3.8626(0.0000)$ & $-3.8628(0.0000)$ & 0.0025 & $-3.8628(0.0000)$ & 0.0025 \\
\hline 4 & Shekel $(4,5)$ & $-10.1526(0.0004)$ & $-10.1532(0.0000)$ & 0.0008 & $-10.0038(0.7469)$ & 248.8052 \\
\hline 4 & Shekel $(4,7)$ & $-10.4019(0.0008)$ & $-10.4029(0.0000)$ & 0.0005 & $-10.3264(0.7612)$ & 76.4323 \\
\hline 4 & Shekel $(4,10)$ & $-10.5363(0.0001)$ & $-10.5364(0.0000)$ & 0.0050 & $-10.5364(0.0000)$ & 0.0050 \\
\hline 5 & Rosenbrock(5) & $0.4324(1.2299)$ & $0.0000(0.0000)$ & $1.16 \mathrm{E}-6$ & $0.0000(0.0000)$ & $1.16 \mathrm{E}-6$ \\
\hline 5 & Zakharov(5) & $0.0000(0.0000)$ & $0.0000(0.0000)$ & 1.0000 & $0.0000(0.0000)$ & 1.0000 \\
\hline 6 & $\operatorname{Hartmann}(6)$ & $-3.3150(0.0283)$ & $-3.3224(0.0000)$ & $6.76 \mathrm{E}-5$ & $-3.3224(0.0000)$ & $6.76 \mathrm{E}-5$ \\
\hline 10 & Sum-Squares(10) & $0.0000(0.0000)$ & $0.0000(0.0000)$ & 1.0000 & $0.0000(0.0000)$ & 1.0000 \\
\hline 10 & Sphere(10) & $0.0000(0.0000)$ & $0.0000(0.0000)$ & 1.0000 & $0.0000(0.0000)$ & 1.0000 \\
\hline 10 & Rosenbrock(10) & $0.9568(1.7026)$ & $0.1595(0.7812)$ & 0.1667 & $0.0000(0.0000)$ & $5.23 \mathrm{E}-7$ \\
\hline 10 & Rastrigin(10) & $4.9748(2.7066)$ & $0.7464(0.8367)$ & 0.1500 & $0.3283(0.4667)$ & 0.0660 \\
\hline 10 & Griewank(10) & $0.0532(0.0310)$ & $0.0474(0.0266)$ & 0.8915 & $0.0426(0.0184)$ & 0.8002 \\
\hline 10 & Zakharov(10) & $0.0000(0.0000)$ & $0.0000(0.0000)$ & 1.0000 & $0.0000(0.0000)$ & 1.0000 \\
\hline 20 & Sphere(20) & $0.0000(0.0000)$ & $0.0000(0.0000)$ & 1.0000 & $0.0000(0.0000)$ & 1.0000 \\
\hline 20 & Rosenbrock(20) & $3.9481(15.1928)$ & $0.4788(1.2955)$ & 0.1213 & $0.0013(0.0078)$ & 0.0003 \\
\hline 20 & Rastrigin(20) & $24.9071(6.7651)$ & $6.8868(3.0184)$ & 0.2765 & $0.7960(1.2833)$ & 0.0319 \\
\hline 20 & Griewank(20) & $0.0129(0.0137)$ & $0.0128(0.0130)$ & 0.9910 & $0.0202(0.0195)$ & 1.5682 \\
\hline 20 & Zakharov(20) & $0.0000(0.0000)$ & $0.0000(0.0000)$ & 1.0000 & $0.0000(0.0000)$ & 1.0000 \\
\hline 30 & Sphere(30) & $0.0000(0.0000)$ & $0.0000(0.0000)$ & 1.0000 & $0.0000(0.0000)$ & 1.0000 \\
\hline 30 & Rosenbrock(30) & $8.6635(6.7336)$ & $0.3627(1.1413)$ & 0.0419 & $0.0632(0.0629)$ & 0.0073 \\
\hline 30 & Rastrigin(30) & $45.1711(15.8998)$ & $11.9425(3.9591)$ & 0.2644 & $1.4327(3.2848)$ & 0.0317 \\
\hline 30 & Griewank(30) & $0.0134(0.0185)$ & $0.0052(0.0080)$ & 0.3907 & $0.0187(0.0163)$ & 1.3980 \\
\hline 30 & Zakharov(30) & $0.9086(4.8932)$ & $0.0000(0.0000)$ & $5.5 \mathrm{E}-7$ & $0.0000(0.0000)$ & $5.5 \mathrm{E}-7$ \\
\hline
\end{tabular}




\subsubsection{Experiment 2}

In the second experiment, we compare $\mathrm{C} /$ CyberSA with the Standard PSO 2007 and the PSwarn algorithm (Vaz \& Vicente, 2007) with an extended set of test functions. The original set from Vaz and Vicente contains 122 test functions, although global optimum solutions were not identified for twelve of these to enable algorithmic performance to be evaluated in these cases. We thus solve the remaining 110 test functions by reference to the experimental setting used in Vaz and Vicente (2007). Thirty runs are executed for each competing algorithm, recording the number of function evaluations consumed when reaching the specified gap to the global optimum, with the limitation that the maximum number of function evaluations for each run is set to 10,000 . For each test function, the best result (in terms of the number of function evaluations) obtained among the 30 runs is reported.

Table 2 lists the specified gap and the best result. The value in parentheses indicates the number of times among the 30 runs that the function value obtained by the algorithm reaches the specified gap. (The result for PSwarm does not include this success rate information because it was not provided in the original paper.) Overall, there are twelve test functions containing either 114 , or 225 , or 294 variables, which is extremely large by the usual standards for global function optimization. In these challenging cases all the competing algorithms fail to obtain a within-gap function value within 10,000 function evaluations. The function value finally obtained is marked with an asterisk $(*)$ and is reported under the column of the corresponding algorithm. For these large and challenging test functions, the $\mathrm{C} / \mathrm{CyberSA}$ method obtains the best function values with the same maximal number of function evaluations as the other methods. The Standard PSO 2007 ranks in second position while the PSwarm obtains the worst objective values for these functions. For the remaining 98 test functions which contain no more than 30 variables, we compare the number of test functions for each of the competing algorithms where the algorithm is unable to reach the specified gap within 10,000 function evaluations. Table 2 shows that the Standard PSO 2007 is less efficient than the other two methods and it fails to solve 17 test functions with satisfactory function values within the 10,000 function evaluation limit. PSwarm performs somewhat better by failing to reach the gap for only 13 test functions and the $\mathrm{C} / \mathrm{CyberSA}$ method performs the best by solving all but 12 test functions.

Next we compare the efficiency for the test functions where the three competing methods can successfully solve to reaching the gap. We consider the number of function evaluations divided by 10,000 (excluding the cases where the algorithm fails to solve the test function) as the probability $p_{i}$ that the corresponding algorithm fails to efficiently solve the test function $i$. Then the geometric mean of the efficiency probability $\left(1-p_{i}\right)$ over the successfully solved cases can be derived by:

$$
\sqrt[|S|]{\prod_{i \in S}\left(1-p_{i}\right)}
$$

where $S$ denotes the set of test functions where the corresponding algorithm successfully reaches the gap. We obtain the efficiency probability for the C/CyberSA, PSwarm, and Standard PSO 2007 as being $98.91 \%, 88.60 \%$, and $83.23 \%$, respectively, thus disclosing that the $\mathrm{C} / \mathrm{CyberSA}$ is able to solve a wider range of test functions more efficiently than the other two algorithms. Moreover, the result also discloses that the PSwarm ranks as more efficient than the Standard PSO 2007 for this set of test functions.

Finally, we compare the success rate (the ratio of the 30 runs that the obtained function value reaches the specified gap) for the Standard PSO 2007 and the C/CyberSA. The success rate for the PSwarm is not available from its original paper. By excluding the failure cases where none of the 30 runs produces a solution that satisfies the gap, the overall success rate can be estimated by the geometric mean of individual rates. The overall success rate for 
Table 2. Number of function evaluations to reach the specified gap to the global optimum by the competing algorithms

\begin{tabular}{|c|c|c|c|c|c|}
\hline$R$ & $\begin{array}{c}\text { Test } \\
\text { Function }\end{array}$ & gap & $\begin{array}{c}\text { Standard PSO } \\
2007\end{array}$ & PSwarm & C/CyberSA \\
\hline 10 & ack & $2.171640 \mathrm{E}-01$ & $10000(0)$ & 1797 & $84(27)$ \\
\hline 2 & ap & $8.600000 \mathrm{E}-05$ & $440(30)$ & 207 & $200(8)$ \\
\hline 2 & bf1 & $0.000000 \mathrm{E}+00$ & $2560(30)$ & 204 & $86(30)$ \\
\hline 2 & bf 2 & $0.000000 \mathrm{E}+00$ & $2240(30)$ & 208 & $88(30)$ \\
\hline 2 & bhs & $1.384940 \mathrm{E}-01$ & $80(30)$ & 218 & $80(30)$ \\
\hline 2 & bl & $0.000000 \mathrm{E}+00$ & $1000(30)$ & 217 & $84(30)$ \\
\hline 2 & $\mathrm{bp}$ & $0.000000 \mathrm{E}+00$ & $1440(30)$ & 224 & $84(30)$ \\
\hline 2 & $\mathrm{cb} 3$ & $0.000000 \mathrm{E}+00$ & 1040(30) & 190 & $84(30)$ \\
\hline 2 & cb6 & $2.800000 \mathrm{E}-05$ & $10000(0)$ & 211 & $10000(0)$ \\
\hline 2 & $\mathrm{~cm} 2$ & $0.000000 \mathrm{E}+00$ & $1160(30)$ & 182 & $159(30)$ \\
\hline 4 & $\mathrm{~cm} 4$ & $0.000000 \mathrm{E}+00$ & $2000(30)$ & 385 & $84(30)$ \\
\hline 2 & da & $4.816600 \mathrm{E}-01$ & $2720(30)$ & 232 & $84(30)$ \\
\hline 10 & em_10 & $1.384700 \mathrm{E}+00$ & $10000(0)$ & 4488 & $10000(0)$ \\
\hline 5 & em_5 & $1.917650 \mathrm{E}-01$ & $4480(2)$ & 823 & $130(4)$ \\
\hline 2 & ep & $0.000000 \mathrm{E}+00$ & $2320(30)$ & 227 & $260(30)$ \\
\hline 10 & $\exp$ & $0.000000 \mathrm{E}+00$ & $3240(30)$ & 1434 & $84(30)$ \\
\hline 2 & fls & $3.000000 \mathrm{E}-06$ & $10000(0)$ & 227 & $10000(0)$ \\
\hline 2 & fr & $0.000000 \mathrm{E}+00$ & $1160(29)$ & 337 & $84(30)$ \\
\hline 10 & $\mathrm{fx} \_10$ & $8.077291 \mathrm{E}+00$ & $10000(0)$ & 1773 & $10000(0)$ \\
\hline 5 & fx_5 & $6.875980 \mathrm{E}+00$ & $440(1)$ & 799 & $10000(0)$ \\
\hline 2 & gp & $0.000000 \mathrm{E}+00$ & $1840(30)$ & 190 & $163(30)$ \\
\hline 3 & grp & $0.000000 \mathrm{E}+00$ & $280(30)$ & 1339 & $135(30)$ \\
\hline 10 & gw & $0.000000 \mathrm{E}+00$ & $10000(0)$ & 2296 & $10000(0)$ \\
\hline 3 & h3 & $0.000000 \mathrm{E}+00$ & $1280(30)$ & 295 & $156(30)$ \\
\hline 6 & h6 & $0.000000 \mathrm{E}+00$ & $2680(14)$ & 655 & $10000(0)$ \\
\hline 2 & $\mathrm{hm}$ & $0.000000 \mathrm{E}+00$ & $1520(30)$ & 195 & $84(30)$ \\
\hline 1 & $\mathrm{hm} 1$ & $0.000000 \mathrm{E}+00$ & $120(30)$ & 96 & $84(30)$ \\
\hline 1 & $\mathrm{hm} 2$ & $1.447000 \mathrm{E}-02$ & $80(30)$ & 141 & $80(30)$ \\
\hline 1 & $\mathrm{hm} 3$ & $2.456000 \mathrm{E}-03$ & $80(30)$ & 110 & $80(30)$ \\
\hline 2 & $\mathrm{hm} 4$ & $0.000000 \mathrm{E}+00$ & $1480(30)$ & 198 & $84(30)$ \\
\hline 3 & $\mathrm{hm} 5$ & $0.000000 \mathrm{E}+00$ & $960(30)$ & 255 & $159(30)$ \\
\hline 2 & hsk & $1.200000 \mathrm{E}-05$ & $120(30)$ & 204 & $170(30)$ \\
\hline 3 & hv & $0.000000 \mathrm{E}+00$ & $2560(30)$ & 343 & $84(30)$ \\
\hline 4 & ir0 & $0.000000 \mathrm{E}+00$ & $5280(30)$ & 671 & $114(27)$ \\
\hline 3 & ir 1 & $0.000000 \mathrm{E}+00$ & $1480(30)$ & 292 & $90(30)$ \\
\hline
\end{tabular}


Table 2. continued

\begin{tabular}{|c|c|c|c|c|c|}
\hline 2 & ir2 & $1.000000 \mathrm{E}-06$ & $1680(30)$ & 522 & $144(30)$ \\
\hline 5 & ir3 & $0.000000 \mathrm{E}+00$ & $320(30)$ & 342 & $172(30)$ \\
\hline 30 & ir4 & $1.587200 \mathrm{E}-02$ & $560(30)$ & 8769 & $84(30)$ \\
\hline 4 & $\mathrm{kl}$ & $4.800000 \mathrm{E}-07$ & $680(30)$ & 1435 & $148(30)$ \\
\hline 1 & ks & $0.000000 \mathrm{E}+00$ & $80(30)$ & 92 & $80(30)$ \\
\hline 114 & 1j1_38 & $4.000000 \mathrm{E}-07$ & $-65.83 *$ & $140.92 *$ & $-83.13^{*}$ \\
\hline 225 & lj1_75 & $4.000000 \mathrm{E}-07$ & $18838.57 *$ & $35129.64 *$ & $5958.58^{*}$ \\
\hline 294 & 1j1_98 & $4.000000 \mathrm{E}-07$ & $134854.88^{*}$ & $193956.8 *$ & $35613.6^{*}$ \\
\hline 114 & lj2_38 & $4.000000 \mathrm{E}-07$ & $146.48 *$ & $372.77 *$ & $161.2^{*}$ \\
\hline 225 & $1 \mathrm{j} 2 \_75$ & $4.000000 \mathrm{E}-07$ & $25227.97 *$ & $32450.09 *$ & $8302.73 *$ \\
\hline 294 & 1j2_98 & $4.000000 \mathrm{E}-07$ & $112291.68^{*}$ & $170045.2 *$ & $52087.07 *$ \\
\hline 114 & 1j3_38 & $4.000000 \mathrm{E}-07$ & $588.51 *$ & $1729.29 *$ & $283.24 *$ \\
\hline 225 & $1 \mathrm{j} 3 \_75$ & $4.000000 \mathrm{E}-07$ & $499130 *$ & $1036894 *$ & $118721^{*}$ \\
\hline 294 & 1j3_98 & $4.000000 \mathrm{E}-07$ & $7667493 *$ & $15188010^{*}$ & $2562334 *$ \\
\hline 3 & $\operatorname{lm} 1$ & $0.000000 \mathrm{E}+00$ & $1760(30)$ & 335 & $84(30)$ \\
\hline 10 & $\operatorname{lm} 2 \_10$ & $0.000000 \mathrm{E}+00$ & $4920(28)$ & 1562 & $162(30)$ \\
\hline 5 & $1 \mathrm{~m} 2 \_5$ & $0.000000 \mathrm{E}+00$ & $2640(30)$ & 625 & $84(30)$ \\
\hline 3 & $\operatorname{lv} 8$ & $0.000000 \mathrm{E}+00$ & $1560(30)$ & 310 & $84(30)$ \\
\hline 2 & $\mathrm{mc}$ & $7.700000 \mathrm{E}-05$ & $160(30)$ & 211 & $84(30)$ \\
\hline 4 & $\mathrm{mcp}$ & $0.000000 \mathrm{E}+00$ & $200(30)$ & 248 & $164(30)$ \\
\hline 2 & mgp & $2.593904 \mathrm{E}+00$ & $80(30)$ & 193 & $80(30)$ \\
\hline 10 & mgw_10 & $1.107800 \mathrm{E}-02$ & $240(30)$ & 10007 & $173(30)$ \\
\hline 2 & mgw_2 & $0.000000 \mathrm{E}+00$ & $80(30)$ & 339 & $84(30)$ \\
\hline 20 & mgw_20 & $5.390400 \mathrm{E}-02$ & $560(27)$ & 10005 & 133(28) \\
\hline 10 & ma_10 & $0.000000 \mathrm{E}+00$ & $10000(0)$ & 2113 & $10000(0)$ \\
\hline 5 & ml_5 & $0.000000 \mathrm{E}+00$ & $2640(8)$ & 603 & $135(15)$ \\
\hline 3 & $\mathrm{mr}$ & $1.860000 \mathrm{E}-03$ & $560(27)$ & 886 & $84(30)$ \\
\hline 2 & mrp & $0.000000 \mathrm{E}+00$ & $1720(18)$ & 217 & $185(24)$ \\
\hline 4 & $\mathrm{nf} 2$ & $2.700000 \mathrm{E}-05$ & $320(30)$ & 2162 & $156(30)$ \\
\hline 10 & nf3_10 & $0.000000 \mathrm{E}+00$ & $10000(0)$ & 4466 & $86(23)$ \\
\hline 15 & nf3_15 & $7.000000 \mathrm{E}-06$ & $10000(0)$ & 10008 & $90(18)$ \\
\hline 20 & nf3_20 & $2.131690 \mathrm{E}-01$ & $10000(0)$ & 10008 & $85(19)$ \\
\hline 25 & nf3_25 & $5.490210 \mathrm{E}-01$ & $10000(0)$ & 10025 & $94(9)$ \\
\hline 30 & nf3_30 & $6.108021 \mathrm{E}+01$ & $10000(0)$ & 10005 & $98(14)$ \\
\hline 10 & osp_10 & $1.143724 \mathrm{E}+00$ & $480(19)$ & 1885 & $132(11)$ \\
\hline 20 & osp_20 & $1.143833 \mathrm{E}+00$ & $80(30)$ & 5621 & $80(30)$ \\
\hline 114 & plj_38 & $4.000000 \mathrm{E}-07$ & $486.37 *$ & $774.64 *$ & $299.12 *$ \\
\hline 225 & plj_75 & $4.000000 \mathrm{E}-07$ & $21733.21 *$ & $37284.11^{*}$ & $10953.88^{*}$ \\
\hline
\end{tabular}


Table 2. continued

\begin{tabular}{|c|c|c|c|c|c|}
\hline 294 & plj_98 & $4.000000 \mathrm{E}-07$ & $111878.79 *$ & $179615.0^{*}$ & $39135.69^{*}$ \\
\hline 10 & $\mathrm{pp}$ & $4.700000 \mathrm{E}-04$ & $2320(30)$ & 1578 & $84(30)$ \\
\hline 2 & prd & $0.000000 \mathrm{E}+00$ & $1440(26)$ & 400 & $126(30)$ \\
\hline 9 & $\mathrm{ptm}$ & $3.908401 \mathrm{E}+00$ & $4280(2)$ & 10009 & $10000(0)$ \\
\hline 4 & pwq & $0.000000 \mathrm{E}+00$ & $2880(30)$ & 439 & $84(30)$ \\
\hline 10 & $\mathrm{rb}$ & $1.114400 \mathrm{E}-02$ & $10000(0)$ & 10003 & $84(16)$ \\
\hline 10 & rg_10 & $0.000000 \mathrm{E}+00$ & $10000(0)$ & 4364 & $170(9)$ \\
\hline 2 & rg_2 & $0.000000 \mathrm{E}+00$ & $1120(29)$ & 210 & $84(30)$ \\
\hline 4 & s10 & $4.510000 \mathrm{E}-03$ & $1560(12)$ & 431 & $84(30)$ \\
\hline 4 & s5 & $3.300000 \mathrm{E}-03$ & $1480(9)$ & 395 & $10000(0)$ \\
\hline 4 & s7 & $3.041000 \mathrm{E}-03$ & $1400(17)$ & 415 & $84(30)$ \\
\hline 10 & sal_10 & $3.998730 \mathrm{E}-01$ & $1400(30)$ & 1356 & $84(30)$ \\
\hline 5 & sal_5 & $1.998730 \mathrm{E}-01$ & $800(30)$ & 452 & $85(30)$ \\
\hline 2 & $\mathrm{sbt}$ & $9.000000 \mathrm{E}-06$ & $1480(30)$ & 305 & $129(30)$ \\
\hline 2 & sfl & $9.716000 \mathrm{E}-03$ & $320(30)$ & 210 & $84(30)$ \\
\hline 2 & $\mathrm{sf} 2$ & $5.383000 \mathrm{E}-03$ & $3280(30)$ & 266 & $90(30)$ \\
\hline 1 & shv1 & $1.000000 \mathrm{E}-03$ & $80(30)$ & 101 & $80(30)$ \\
\hline 2 & shv2 & $0.000000 \mathrm{E}+00$ & $640(30)$ & 196 & $154(30)$ \\
\hline 10 & $\sin \_10$ & $0.000000 \mathrm{E}+00$ & $4560(29)$ & 1872 & $85(30)$ \\
\hline 20 & $\sin \_20$ & $0.000000 \mathrm{E}+00$ & $8360(25)$ & 5462 & $84(29)$ \\
\hline 17 & st_17 & $3.081935 \mathrm{E}+06$ & $4160(5)$ & 10011 & $127(30)$ \\
\hline 9 & st_9 & $7.516622 \mathrm{E}+00$ & $10000(0)$ & 10001 & $10000(0)$ \\
\hline 1 & stg & $0.000000 \mathrm{E}+00$ & $80(30)$ & 113 & $84(30)$ \\
\hline 10 & swf & $1.184385 \mathrm{E}+02$ & $10000(0)$ & 2311 & $10000(0)$ \\
\hline 1 & sz & $2.561249 \mathrm{E}+00$ & $80(30)$ & 125 & $80(29)$ \\
\hline 1 & sZZs & $1.308000 \mathrm{E}-03$ & $80(30)$ & 112 & $80(30)$ \\
\hline 4 & wf & $2.500000 \mathrm{E}-05$ & $10000(0)$ & 10008 & $84(17)$ \\
\hline 10 & zkv_10 & $1.393000 \mathrm{E}-03$ & $3920(30)$ & 10003 & $84(30)$ \\
\hline 2 & zkv_2 & $0.000000 \mathrm{E}+00$ & $1120(30)$ & 212 & $84(30)$ \\
\hline 20 & zkv_20 & $3.632018 \mathrm{E}+01$ & $840(29)$ & 10018 & 171(30) \\
\hline 5 & zkv_5 & $0.000000 \mathrm{E}+00$ & $3280(30)$ & 1318 & $84(30)$ \\
\hline 1 & zlk1 & $4.039000 \mathrm{E}-03$ & $200(30)$ & 119 & $126(30)$ \\
\hline 1 & zlk2a & $5.000000 \mathrm{E}-03$ & $80(30)$ & 130 & $80(30)$ \\
\hline 1 & zlk2b & $5.000000 \mathrm{E}-03$ & $80(30)$ & 113 & $80(30)$ \\
\hline 2 & zlk4 & $2.112000 \mathrm{E}-03$ & $240(30)$ & 224 & $162(9)$ \\
\hline 3 & zlk5 & $2.782000 \mathrm{E}-03$ & $200(30)$ & 294 & $166(5)$ \\
\hline 1 & zzs & $4.239000 \mathrm{E}-03$ & $80(30)$ & 120 & $80(23)$ \\
\hline
\end{tabular}


the Standard PSO 2007 and the C/CyberSA is derived according to the numbers listed in parentheses in Table 2, being $80.98 \%$ and $84.95 \%$, respectively. In addition to solving five more test functions than the Standard PSO 2007, the C/CyberSA also manifests a higher success rate for the successfully solved cases. Consequently the C/CyberSA is more effective than the Standard PSO 2007 on all measures.

\section{CONCLUDING REMARKS}

Our Complementary Cyber Swarm Algorithm (C/CyberSA) draws on the basic principles underlying the original CyberSA method, but adopts different sets of ideas from tabu search (TS) and scatter search/path relinking (SS/ PR). Extensive empirical tests with a set of 110 test functions shows that the C/CyberSA can produce improvements comparable to those provided by the CyberSA in relation to PSO methods that do not incorporate such ideas. The C/CyberSA exploits guidance and restriction information derived by applying adaptive memory strategies from TS to the history of swarm search and incorporates path relinking as an essential component to yield two longterm strategies as responses to the detection of critical events encountered in the search.

Our experimental results show that the C/CyberSA outperforms the PSwarm and the Standard PSO 2007 methods by finding more optimal solutions of the test problems and by simultaneously using a smaller number of function evaluations. In addition, we find that a "trivial combination" of C/CyberSA and CyberSA that runs for 250,000 function evaluations strongly dominates the Standard PSO 2007 method when the latter method is allotted this number of function evaluations, obtaining better solutions on 16 out of 30 basic test cases and matching the quality of solutions obtained by the Standard PSO 2007 on the remaining 14 cases. These findings motivate future investigations of Cyber Swarm methods that combine features of both the original and complementary variants and incorporate additional strategic notions from SS and PR.

\section{REFERENCES}

Angeline, P. J. (1999). Using selection to improve particle swarm optimization. In Proceedings of the IEEE International Joint Conference on Neural Networks (pp. 84-89).

Audet, C., \& Dennis, J.E. (2003). Analysis of generalized pattern searches. SIAM Journal on Optimization, 13, 889-903. doi:10.1137/S1052623400378742

Campos, V., Glover, F., Laguna, M., \& Martí, R. (2001). An experimental evaluation of a scatter search for the linear ordering problem. Journal of Global Optimization, 21(4), 397-414. doi:10.1023/A:1012793906010

Chelouah, R., \& Siarry, P. (2000). Tabu search applied to global optimization. European Journal of Operational Research, 123, 256-270. doi:10.1016/ S0377-2217(99)00255-6

Chelouah, R., \& Siarry, P. (2005). A hybrid method combining continuous tabu search and Nelder-Mead simplex algorithms for the global optimization of multiminima functions. European Journal of $\mathrm{Op}$ erational Research, 161, 636-654. doi:10.1016/j. ejor.2003.08.053

Clerc, M. (2008). Particle swarm programs. Retrieved from http://www.particleswarm.info/ Programs.html

Clerc, M., \& Kennedy, J. (2002). The particle swarm explosion, stability, and convergence in a multidimensional complex space. IEEE Transactions on Evolutionary Computation, 6, 58-73. doi:10.1109/4235.985692

Duarte, A., Marti, R., \& Glover, F. (2007). Adaptive memory programming for global optimization. Valencia, Spain: University of Valencia.

Duarte, A., Marti, R., \& Glover, F. (2011a). Hybrid scatter-tabu search for unconstrained global optimization. Annals of Operations Research, 183, 95-123. doi:10.1007/s10479-009-0596-2

Duarte, A., Marti, R., \& Glover, F. (2011b). Path relinking for large scale global optimization. Soft Computing, 15.

Eberhart, R. C., \& Shi, Y. (1998). Evolving artificial neural networks. In Proceedings of the International Conference on Neural Networks and Brain (pp. 5-13).

Feng, H. M., Chen, C. Y., \& Ye, F. (2007). Evolutionary fuzzy particle swarm optimization vector quantization learning scheme in image compression. Expert Systems with Applications, 32, 213-222. doi:10.1016/j.eswa.2005.11.012 
Glover, F. (1986). Future paths for integer programming and links to artificial intelligence. Computers \& Operations Research, 13, 533-549. doi:10.1016/0305-0548(86)90048-1

Glover, F. (1994). Tabu search for nonlinear and parametric optimization (with links to genetic algorithms). Discrete Applied Mathematics, 49, 231-255. doi:10.1016/0166-218X(94)90211-9

Glover, F. (1996a). Tabu search and adaptive memory programming - advances, applications and challenges. In Barr, R. S., Helgason, R. V., \& Kennington, J. L. (Eds.), Interfaces in computer science and operations research (pp. 1-75). Boston, MA: Kluwer Academic.

Glover, F. (1996b). Ejection chains, reference structures and alternating path methods for traveling salesman problems. Discrete Applied Mathematics, 65, 223-253. doi:10.1016/0166-218X(94)00037-E

Glover, F. (1998). A template for scatter search and path relinking. In J.-K. Hao, E. Lutton, E. M. A. Ronald, M. Schoenauer, \& D. Snyers (Eds.), Proceedings of the Third European Conference on Artificial Evolution (LNCS 1363, pp. 3-54).

Glover, F., \& Laguna, M. (1997). Tabu search. Boston, MA: Kluwer Academic.

Hedar, A., \& Fukushima, M. (2006). Tabu search directed by direct search methods for nonlinear global optimization. European Journal of Operational Research, 170(2), 329-349. doi:10.1016/j. ejor.2004.05.033

Hirsch, M. J., Meneses, C. N., Pardalos, P. M., \& Resende, M. G. C. (2007). Global optimization by continuous grasp. Optimization Letters, 1(2), 201-212. doi:10.1007/s11590-006-0021-6

Ho, S. C., \& Gendreau, M. (2006). Path relinking for the vehicle routing problem. Journal of Heuristics, 12, 55-72. doi:10.1007/s10732-006-4192-1

Hvattum, L. M., \& Glover, F. (2009). Finding local optima of high-dimensional functions using direct search methods. European Journal of Operational Research, 195, 31-45. doi:10.1016/j. ejor.2008.01.039
James, T., Rego, C., \& Glover, F. (2009). Multistart tabu search and diversification strategies for the quadratic assignment problem. IEEE Transactions on Systems, Man, and Cybernetics. Part A, Systems and Humans, 39(3). doi:10.1109/TSMCA.2009.2014556

Kennedy, J. (1999, July). Small world and megaminds: Effects of neighbourhood topology on particle swarm performance. In Proceedings of the Congress on Evolutionary Computation (pp. 1931-1938).

Kennedy, J., \& Eberhart, R. C. (1995). Particle swarm optimization. In Proceedings of the IEEE International Conference on Neural Networks, IV, 1942-1948. doi:10.1109/ICNN.1995.488968

Laguna, M., \& Marti, R. (2003). Scatter search: Methodology and implementation in C. Boston, MA: Kluwer Academic.

Laguna, M., \& Marti, R. (2005). Experimental testing of advanced scatter search designs for global optimization of multimodal functions. Journal of Global Optimization, 33, 235-255. doi:10.1007/ s10898-004-1936-z

Lepagnot, J., Nakib, A., Oulhadj, H., \& Siarry, P. (2010). A new multiagent algorithm for dynamic continuous optimization. International Journal of Applied Metaheuristic Computing, 1(1), 16-38. doi:10.4018/jamc.2010102602

Lovbjerg, M., Rasmussen, T. K., \& Krink, T. (2001). Hybrid particle swarm optimizer with breeding and subpopulations. In Proceedings of the Genetic and Evolutionary Computation Conference.

Lu,Z., Hao, J.-K., \& Glover, F. (2010). Neighborhood analysis: A case study on curriculum-based course timetabling. Journal of Heuristics.

Luke, S. (2009). Essentials of metaheuristics. Retrieved from http://cs.gmu.edu/ sean/book/ metaheuristics/

Mendes, R., Kennedy, J., \& Neves, J. (2004). The fully informed particle swarm: Simpler, maybe better. IEEE Transactions on Evolutionary Computation, , 204-210. doi:10.1109/TEVC.2004.826074

Miranda, V., Keko, H., \& Jaramillo, A. (2007). EPSO: Evolutionary particle swarms. Studies in Computational Intelligence, 66, 139-167. doi:10.1007/9783-540-72377-6_6 
Mladenovic, N., \& Hansen, P. (1997). Variable neighborhood search. Computers \& Operations Research, 24, 1097-1100. doi:10.1016/S03050548(97)00031-2

Nakano, S., Ishigame, A., \& Yasuda, K. (2007). Particle swarm optimization based on the concept of tabu search. In Proceedings of the IEEE Congress on Evolutionary Computation (pp. 3258-3263).

Rego, C., \& Glover, F. (2009). Ejection chain and filter-and-fan methods in combinatorial optimization. Annals of Operations Research, 175(1), 77-105. doi:10.1007/s10479-009-0656-7

Shen, Q., Shi, W. M., \& Kong, W. (2008). Hybrid particle swarm optimization and tabu search approach for selecting genes for tumor classification using gene expression data. Computational Biology and Chemistry, 32(1), 52-59. doi:10.1016/j.compbiolchem.2007.10.001

Shigenori, N., Takamu, G., Toshiku, Y., \& Yoshikazu, F. (2003). A hybrid particle swarm optimization for distribution state estimation. IEEE Transactions on Power Systems, 18, 60-68. doi:10.1109/ TPWRS.2002.807051

Sörensen, K., \& Glover, F. (2010). Metaheuristics. In Gass, S., \& Fu, M. (Eds.), Encyclopedia of operations research (3rd ed.). New York, NY: Springer.
Sörensen, K., Sevaux, M., \& Schittekat, P. (2008). Multiple neighbourhood search in commercial VRP packages: Evolving towards self-adaptive methods. Studies in Computational Statistics, 136, 239-253.

Vaz,A. I. F., \& Vicente, L. N. (2007).A particle swarm pattern search method for bound constrained global optimization. Journal of Global Optimization, 39, 197-219. doi:10.1007/s10898-007-9133-5

Wang, Y. X., Zhao, Z. D., \& Ren, R. (2007). Hybrid particle swarm optimizer with tabu strategy for global numerical optimization. In Proceedings of the IEEE Congress on Evolutionary Computation (pp. 2310-2316).

Yin, P. Y., Glover, F., Laguna, M., \& Zhu, J. X. (2010). Cyber swarm algorithms - improving particle swarm optimization using adaptive memory strategies. European Journal of Operational Research, 201(2), 377-389. doi:10.1016/j.ejor.2009.03.035

Yoshida, H., Kawata, K., Fukuyama, Y., \& Nakanishi, Y.(1999). A particle swarm optimization for reactive power and voltage control considering voltage stability. In Proceedings of the International Conference on Intelligent System Application to Power Systems (pp. 117-121).

Peng-Yeng Yin is a Professor of the Department of Information Management, National Chi Nan University, Taiwan, and he is currently the Dean of the Office of Research and Development. From 1993 to 1994, he was a visiting scholar at the Department of Electrical Engineering, University of Maryland, College Park. In 2000, he was a visiting Professor at the Department of Electrical Engineering, University of California, Riverside. From 2006 to 2007, he was a visiting Professor at Leeds School of Business, University of Colorado. Dr. Yin is a member of the Phi Tau Phi Scholastic Honor Society and listed in Who's Who in the World, Who's Who in Science and Engineering, and Who's Who in Asia. He is the Editor-in-Chief of the International Journal of Applied Metaheuristic Computing and is on the Editorial Board of International Journal of Advanced Robotic Systems, Journal of Education, Informatics and Cybernetics, The Open Artificial Intelligence Journal, The Open Signal Processing Journal and served as a program committee member in many international conferences. He has also edited two books in the pattern recognition area. His current research interests include artificial intelligence, evolutionary computation, metaheuristics, pattern recognition, machine learning, and operations research. 
Fred Glover is the Chief Technology Officer in charge of algorithmic design and strategic planning initiatives for OptTek Systems, Inc., heading the development of commercial computer software systems currently serving more than 80,000 users in the United States and abroad. He also holds the title of Distinguished Professor at the University of Colorado, Boulder, where he is affiliated with the Leeds School of Business and the Department of Electrical and Computer Engineering. Dr. Glover is widely known for his work in the applications of computer decision support systems, including industrial planning, financial analysis, systems design, energy and natural resources planning, logistics, transportation and large-scale allocation models. He has authored or co-authored more than 400 published articles and eight books in the fields of mathematical optimization, computer science and artificial intelligence. He is also the originator of Tabu Search (Adaptive Memory Programming), an optimization search methodology of which more than 200,000 Web pages can be found with a simple Google search. Glover is the recipient of the highest honor of the Institute of Operations Research and Management Science, the von Neumann Theory Prize, and is an elected member of the National Academy of Engineering. He has also received numerous other awards and honorary fellowships, including those from the American Association for the Advancement of Science (AAAS), the NATO Division of Scientific Affairs, the Institute of Operations Research and Management Science (INFORMS), the Decision Sciences Institute (DSI), the U.S. Defense Communications Agency (DCA), the Energy Research Institute (ERI), the American Assembly of Collegiate Schools of Business (AACSB), Alpha Iota Delta, the Institute of Cybernetics of the Ukrainian Academy of Science, and the Miller Institute for Basic Research in Science.

Manuel Laguna is the Media One Professor of Management Science and Senior Associate Dean of the Leeds School of Business of the University of Colorado at Boulder. He started his career at the University of Colorado in 1990, after receiving master's (1987) and doctoral (1990) degrees in Operations Research and Industrial Engineering from the University of Texas at Austin. He has done extensive research in the interface between computer science, artificial intelligence and operations research to develop solution methods for practical problems in operations-management areas such as logistics and supply chains, telecommunications, decision-making under uncertainty and optimization of simulated systems. Dr. Laguna has more than one hundred publications, including more than sixty articles in academic journals and four books. He is editor-in-chief of the Journal of Heuristics, is in the international advisory board of the Journal of the Operational Research Society and has been guest editor of the Annals of Operations Research and the European Journal of Operational Research. Dr. Laguna has received research funding from government agencies such as the National Science Foundation, Office of Naval Research and the Environmental Protection Agency. He is member of the Institute for Operations Research and the Management Science and was the general chair of the Institute's national meeting in 2004. He is also member of Beta Gamma Sigma and the International Honor Society Omega Rho. Dr. Laguna is co-founder of OptTek Systems, a Boulder-based software and consulting company that provides optimization solutions.

Jia-Xian Zhu received his B.S. degree in Information Management at National Formosa University, Yunlin, Taiwan in 2006 and the M.B.A. degree in Information Management at National Chi Nan University, Nantou, Taiwan in 2008. He has published an article in European Journal of Operational Research. His research interests include metaheuristics, machine learning, software engineering, and operations research. 